\title{
Secular trend for mortality from breast cancer and the association between diabetes and breast cancer in Taiwan between 1995 and 2006
}

\author{
C.-H. Tseng • C.-K. Chong $\cdot$ T.-Y. Tai
}

Received: 8 August 2008 / Accepted: 16 October 2008 / Published online: 19 November 2008

(C) Springer-Verlag 2008

\begin{abstract}
Aims/hypothesis Studies have identified an association between diabetes and breast cancer in postmenopausal women in Western countries. Such an association needs to be confirmed in an Asian population. The aim of this study was to evaluate the secular trend for breast cancer mortality in Taiwanese women in the general population and the mortality rate ratios between diabetic patients and the general population.

Methods Age-specific mortality rates for the general population, categorised into groups aged 25-54, 55-64, 65-74 and $\geq 75$ years, were calculated for the years between 1995 and 2006 (inclusive) from vital statistics published by the Taiwanese government. Linear regression was used to test the trends. A total of 131,573 diabetic women aged $\geq 25$ years from a national cohort recruited between 1995 and 1998
\end{abstract}

C.-H. Tseng · T.-Y. Tai

Department of Internal Medicine,

National Taiwan University College of Medicine,

Taipei, Taiwan

C.-H. Tseng $(\bowtie) \cdot$ T.-Y. Tai

Division of Endocrinology and Metabolism,

Department of Internal Medicine,

National Taiwan University Hospital,

No. 7 Chung-Shan South Road,

Taipei 10002, Taiwan

e-mail: ccktsh@ms6.hinet.net

C.-H. Tseng

Department of Medical Research and Development,

National Taiwan University Hospital Yun-Lin Branch,

Yun-Lin, Taiwan

C.-K. Chong

Department of Rehabilitation, Chang Gung Memorial Hospital,

Taoyuan, Taiwan (inclusive) were followed prospectively for vital status, determined from the National Register of Deaths. Mortality rates and mortality rate ratios (mortality rate in diabetic women vs the average and highest mortality rates for the general population) were calculated.

Results A total of 14,230 women aged $\geq 25$ years in the general population died of breast cancer between 1995 and 2006. A trend for an increase in the annual rate was observed for all age groups. A total of 482 diabetic women died of breast cancer, with a crude mortality rate of 45.7 per 100,000 person-years. Compared with the general population the relative risk of mortality for those with diabetes ranged from 1.37 (for the group aged 55-64 years) to 2.43 (for the group aged 25-54 years).

Conclusions/interpretation We identified a secular trend of an increase in the rate of breast cancer mortality in the Taiwanese general population. Our data suggest a higher risk of breast cancer mortality in diabetic patients in all age groups.

Keywords Breast cancer · Diabetes · Mortality . Mortality rate ratio $\cdot$ Secular trend

\section{Abbreviations \\ ICD International Classification of Diseases \\ NHI National Health Insurance}

\section{Introduction}

Although the association between breast cancer and diabetes mellitus was first described more than a century ago [1], controversial conclusions on the risk of breast cancer in diabetic patients are found in the literature. For 
example, two recent meta-analyses showed a significant increase of breast cancer risk of $15 \%$ [2] to $20 \%$ [3] in women with diabetes, and suggested that the risk may be consistently higher in postmenopausal women or among women of postmenopausal age [2]. The link between diabetes and breast cancer is also strengthened by the observation that a higher fasting glucose level is associated with a higher risk of breast cancer [4, 5], and by the results of a recent meta-analysis suggesting that dietary glycaemic index is significantly associated with a higher risk of breast cancer [6]. However, not all studies included in the metaanalyses agreed that there is a significantly higher risk of breast cancer in diabetic patients $[2,3]$ or in individuals with a higher dietary glycaemic index [6]. Furthermore, a more recent epidemiological study conducted in the USA did not find an association between diabetes (specifically, a history of diabetes without classification) and breast cancer [7].

Most of the studies evaluating the association between diabetes and breast cancer risk were conducted in the Western world, and data from Asian countries are still scarce. Furthermore, inconsistent results have also been found in Asian populations. In one study, a positive association was observed in Asian-American women, especially those who were low/intermediate soy consumers [8]. A study conducted in Japan [9] and another one in Korea [10] concluded that a significantly higher risk of breast cancer is associated with diabetes, reporting respective ORs of 2.06 (95\% CI 1.27-3.34) and 1.51 (95\% CI 1.26-1.80). However, such an association could not be reconfirmed by two later Japanese studies [11, 12].

One of the studies to investigate the association between diabetes and breast cancer mortality was a 16 year followup study conducted in the USA that recruited 588,321 women without reported cancer at enrollment in 1982 [13]. The investigators found that diabetes is significantly associated with a 1.27 -fold higher risk $(95 \%$ CI 1.11 1.45) of mortality from breast cancer [13]. Similarly, a 1.40 -fold (95\% CI 1.06-1.81) higher risk of mortality from breast cancer was observed in a 10 year follow-up study of 3,782 diabetic women in Italy from 1987 to 1996 [14]. In the 10 year follow-up study conducted in 468,615 Korean women, a significantly higher risk of breast cancer mortality was also observed in the diabetic patients, with an adjusted relative risk of 2.23 (95\% CI 1.49-3.33) [10]. On the other hand, a study conducted in the UK of 28,900 insulin-treated diabetic patients, 23,834 of whom had been diagnosed with diabetes under the age of 30 years (presumably with type 1 diabetes) and 5,066 of whom had been diagnosed between the ages of 30 and 49 years (presumably with type 2 diabetes), did not show a significantly higher risk of breast cancer mortality in either those with type 1 or type 2 diabetes [15].
To the best of our knowledge, no previous study investigating the association between diabetes and breast cancer mortality has ever been conducted in Taiwanese individuals. Furthermore, most previous studies are limited by the fact that they did not differentiate between type 1 and type 2 diabetes [2]. Therefore, the purpose of this study was to evaluate, in groups stratified according to age, (1) the secular trends for mortality from breast cancer in the general population of Taiwan; and (2) the association between diabetes and mortality from breast cancer, in all types of diabetic patients and in patients with type 2 diabetes.

\section{Methods}

Mortality from breast cancer in women of the general population The study was approved and funded by the Department of Health, Executive Yuan, Taiwan. Because breast cancer is very rare among young women, we chose to analyse the data for women aged 25 years or older, who were categorised into the following age groups: $25-54,55$ $64,65-74$ and $\geq 75$ years old. The data for mortality from breast cancer were taken from vital statistics reported annually by the government of Taiwan. Since 1981, causes of death have been coded according to the International Classification of Diseases, ninth revision (ICD-9) in Taiwan. Death as a result of breast cancer was defined according to ICD-9 code 174. Because the contributing causes of death were not available, only the underlying cause of death was used for analyses in this study.

Annual age-specific mortality rates were calculated for each year between 1995 and 2006 (inclusive) using the total number of deaths ascribed to breast cancer for that particular year and the mid-year female population for the year in question for the specific age groups. The average mortality rates for the specific age groups over the 12 year period between 1995 and 2006 were calculated by determining the average annual number of deaths caused by breast cancer and dividing this by the average mid-year population. Linear regression was used to evaluate whether there was a significant change in trend with regard to the annual breast cancer mortality rate over the duration of the study, using the mortality rate as the dependent variable and the calendar year as the independent variable.

Mortality from breast cancer in the diabetic patients In March 1995, a compulsory and universal system of health insurance that covers $>96 \%$ of the total population, known as National Health Insurance (NHI), was implemented in Taiwan. Between 1995 and 1998 (inclusive), using the NHI database, a cohort of 256,036 diabetic patients (referred to as 'the original cohort' in this study) was established for investigating a series of epidemiological issues related to 
diabetes [16]. The establishment of this national cohort of diabetic patients has been described in detail elsewhere [16]. The diagnosis of diabetes was defined by ICD-9 code 250 or the A-code (abridged code) A181. Since the claim data included both inpatients and outpatients, it was believed that this recruitment procedure would not tend to select sicker or healthier patients [16]. Although it is not easy to specify the sensitivity and specificity of this approach, both were believed to be good because the clinical settings would tend to report all cases of diabetes in the claim data to ensure full reimbursement and because a false report of diagnosis would incur a severe penalty from the Bureau of NHI [16].

All the patients were followed until the end of the year 2006. Vital status and the date and cause of death of those who died were obtained by looking up the computerised data file for each participant on the National Register of Deaths using their unique identification number. Mortality rates were calculated using a person-years denominator. The personyears of follow-up for each patient were calculated as the duration from the date of recruitment until the end of the year 2006 for those who were alive or to the date of death for those who died. Age-specific mortality rates and the mortality rate ratios for the diabetic women vs the general female population were calculated. The age-specific mortality rate ratios were calculated using the average mortality rates within the 12 years in the general population as referents. Because we noticed a trend for an increase in the annual rate of mortality from breast cancer in the general population over the duration of the study, the mortality rate ratios for the diabetic patients vs the general population were also calculated using the highest annual mortality rate for each age group in the general population as comparison groups.

For sensitivity analyses, we calculated the mortality rates and the mortality rate ratios in the subgroup of patients who had been interviewed using a baseline questionnaire after receiving their informed consent, as described in detail elsewhere [17-20]. The total number of diabetic patients interviewed was 93,484 , and 49,405 of these were women aged $\geq 25$ years (referred to as 'the subcohort of diabetic women aged $\geq 25$ years'). To evaluate whether the association was found in patients with type 2 diabetes, the mortality rates and mortality rate ratios were also calculated after excluding patients with type 1 diabetes based on the presence of diabetic ketoacidosis at the onset of diabetes or the need for insulin injection within 1 year after diagnosis of diabetes. There were 47,874 diabetic women remaining after the exclusion of those with type 1 diabetes and these women are referred to as 'the subcohort of type 2 diabetic women aged $\geq 25$ years'. Because only 1,531 patients were excluded with a possible diagnosis of type 1 diabetes, only five of whom died of breast cancer, we did not analyse the association with these patients.
All analyses were conducted using SAS statistical software, version 9.1 (SAS Institute, Cary, NC, USA).

\section{Results}

A total of 14,230 women in the general population aged 25 years or older died of breast cancer between 1995 and 2006 in Taiwan according to data obtained from the government vital statistics. Table 1 shows the age-specific mortality rates (per 100,000) from breast cancer in the general population of Taiwan for each year between 1995 and 2006 and the age-specific average annual mortality rates for the 12 year period. The mortality rates increased dramatically with increasing age. A secular trend of an increase in the annual mortality rate from breast cancer over the 12 year period was observed for all age groups $(p<0.05)$.

A total of 131,573 diabetic women aged 25 years or older in the original cohort at recruitment were followed. Among them, 482 died of breast cancer, which equates to a mortality rate of 45.7 per 100,000 person-years (Table 2). The agespecific mortality rates from breast cancer for the diabetic patients and their mortality rate ratios compared with the general Taiwanese population are shown in Table 2. Using either the average mortality rates or the highest mortality rates (i.e. the mortality rate for the year 2003, 2005, 2003 and 2003, respectively, for the groups aged 25-54, 55-64, 65-74 and $\geq 75$ years, as shown in Table 1) for the general population as the referents, the age-specific mortality rate ratios for the original cohort were all significantly different from 1, and was highest for the group aged 25-54 years.

In the sensitivity analyses, using the highest mortality rates in the general population as referents, the mortality rates and mortality rate ratios were similar to those observed for the original cohort, except that the mortality rate ratios in the group aged 55-64 years were not significantly different from 1 (though the ratios still favoured a positive association; Table 2).

\section{Discussion}

The results of this study suggest that the mortality rate from breast cancer in the general population of Taiwan increased for all age groups during the period between 1995 and 2006 (Table 1). This observation is contradictory to what has been observed in the USA, where the overall mortality rate from breast cancer has decreased over the past 15 years [21].

Although there was an increased risk of mortality from breast cancer in the diabetic patients in all age groups compared with the general population, the increase was most remarkable in the group aged 25-54 years (Table 2). If 
Diabetologia (2009) 52:240-246

243

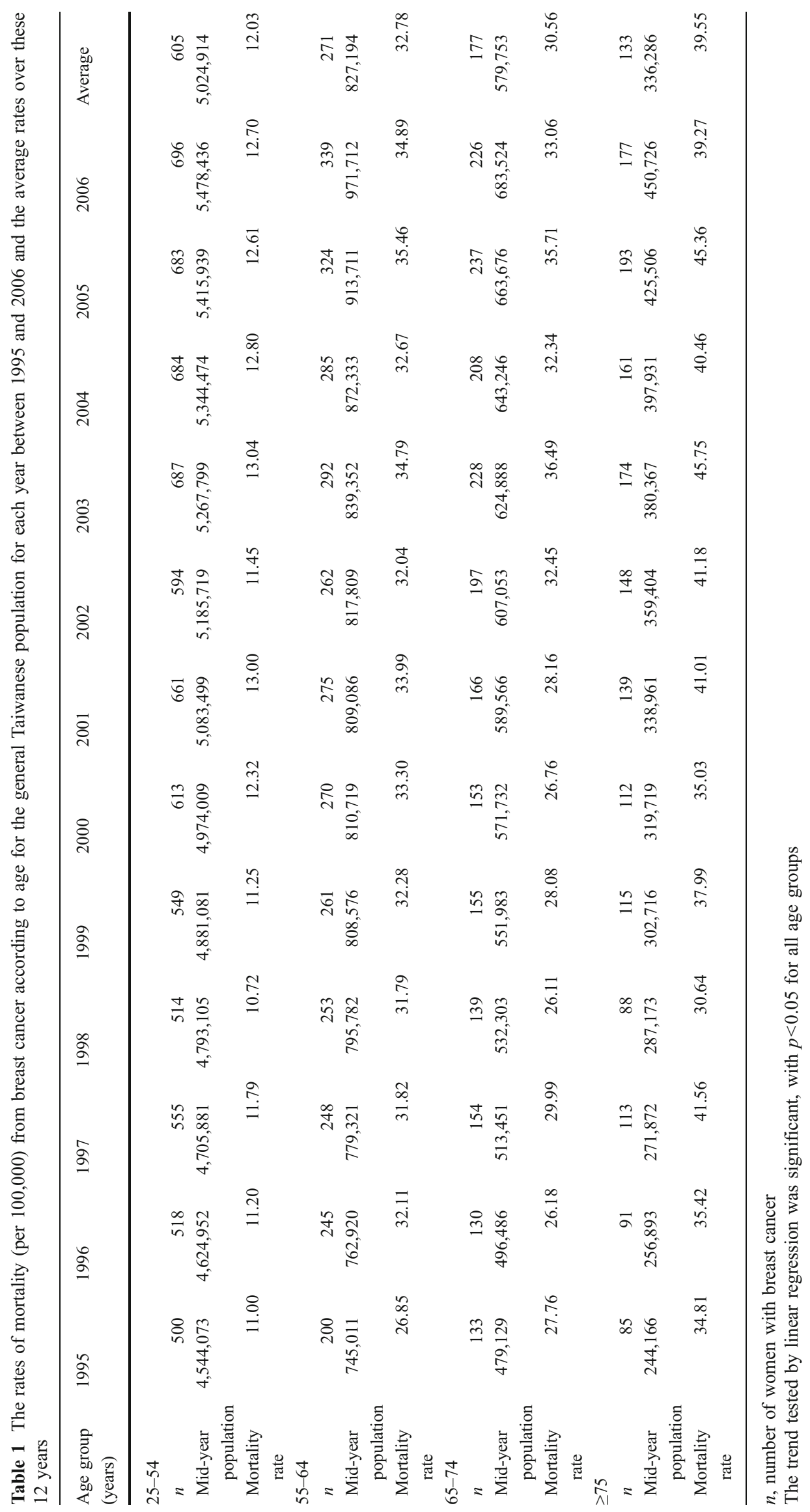

Springer 
Table 2 Age-specific rates of mortality from breast cancer for the diabetic women and their mortality rate ratios compared with the general population in Taiwan

\begin{tabular}{|c|c|c|c|c|c|c|}
\hline Age group (years) & $n$ & $N$ & $\begin{array}{l}\text { Person- } \\
\text { years }\end{array}$ & $\begin{array}{l}\text { Mortality rate } \\
\text { (per } 100,000 \\
\text { person-years) }\end{array}$ & $\begin{array}{l}\text { Mortality rate ratio vs the } \\
\text { average mortality rate for the } \\
\text { general population }(95 \% \mathrm{CI})\end{array}$ & $\begin{array}{l}\text { Mortality rate ratio vs the } \\
\text { highest mortality rate for the } \\
\text { general population }(95 \% \mathrm{CI})\end{array}$ \\
\hline \multicolumn{7}{|c|}{ Original cohort analysis } \\
\hline \multicolumn{7}{|c|}{ Diabetic women aged $\geq 25$ years } \\
\hline $25-54$ & 117 & 43,749 & $399,556.9$ & 29.28 & $2.43(2.04-2.90)$ & $2.25(1.86-2.72)$ \\
\hline $55-64$ & 141 & 37,317 & $314,033.6$ & 44.90 & $1.37(1.16-1.62)$ & $1.27(1.04-1.54)$ \\
\hline $65-74$ & 158 & 35,194 & $258,155.6$ & 61.20 & $2.00(1.71-2.35)$ & $1.68(1.37-2.05)$ \\
\hline$\geq 75$ & 66 & 15,313 & $83,905.5$ & 78.66 & $1.99(1.56-2.53)$ & $1.72(1.30-2.27)$ \\
\hline \multicolumn{7}{|l|}{ Sensitivity analyses } \\
\hline \multicolumn{7}{|c|}{$\begin{array}{l}\text { Subcohort of diabetic women } \\
\text { aged } \geq 25 \text { years }\end{array}$} \\
\hline $25-54$ & 38 & 12,768 & $95,960.8$ & 39.60 & $3.29(2.44-4.45)$ & $3.04(2.23-4.14)$ \\
\hline $55-64$ & 53 & 16,717 & $118,278.5$ & 44.81 & $1.37(1.04-1.79)$ & $1.26(0.95-1.69)$ \\
\hline $65-74$ & 59 & 15,029 & $96,058.4$ & 61.42 & $2.01(1.56-2.59)$ & $1.68(1.27-2.23)$ \\
\hline$\geq 75$ & 24 & 4,891 & $25,384.8$ & 94.54 & $2.39(1.62-3.53)$ & $2.07(1.36-3.14)$ \\
\hline \multicolumn{7}{|c|}{$\begin{array}{l}\text { Subcohort of type } 2 \text { diabetic } \\
\text { women aged } \geq 25 \text { years }\end{array}$} \\
\hline $25-54$ & 36 & 12,260 & $92,076.3$ & 39.10 & $3.25(2.39-4.43)$ & $3.00(2.18-4.12)$ \\
\hline $55-64$ & 52 & 16,288 & $115,494.5$ & 45.02 & $1.37(1.05-1.80)$ & $1.27(0.95-1.70)$ \\
\hline $65-74$ & 57 & 14,570 & $93,223.1$ & 61.14 & $2.00(1.55-2.59)$ & $1.68(1.26-2.23)$ \\
\hline$\geq 75$ & 24 & 4,756 & $24,716.7$ & 97.10 & $2.46(1.66-3.63)$ & $2.12(1.40-3.22)$ \\
\hline
\end{tabular}

$n$, number of women with breast cancer

this age group was to be used as a proxy for premenopausal status, then our results seem to be inconsistent with a metaanalysis that showed a stronger association (overall summary RR 1.19, 95\% CI 1.15-1.23) among postmenopausal women or among women of postmenopausal age and no significant association for premenopausal women or women of premenopausal age [2]. Our results indicated that the association was independent of menopausal status, although the mortality rate ratios may not be significant in the group aged 55-64 years according to the sensitivity analyses that compared the mortality rates for the diabetic patients with the highest mortality rates for the general population (Table 2). One possible explanation for the discrepancies could be the ethnicities of the study populations, because none of the studies analysed according the menopausal status in the meta-analysis [2] had enrolled Asian women.

Three previous studies have shown a significantly higher risk of breast cancer mortality in relation to diabetes in the USA [13], Italy [14] and Korea [10]. Except for the Italian study, which recruited patients with type 2 diabetes [14], the studies conducted in the USA [13] and Korea [10] did not distinguish between type 1 and type 2 diabetes. However, because a greater proportion of diabetic patients have type 2 diabetes, the diabetic patients in both these studies probably mainly represent type 2 diabetes. In the present study we showed that there was an increased risk of breast cancer mortality irrespective of whether all diabetic patients or only patients with type 2 diabetes were included in the analysis (Table 2). We did not evaluate whether there was an association in patients with type 1 diabetes because of the relatively small number of such cases in our cohort. Previously, a higher risk of breast cancer mortality was not observed in 23,834 insulin-treated patients with type 1 diabetes (based on an age of onset of $<30$ years) in the UK [15]. Taken together, the increased risk of breast cancer mortality associated with diabetes probably only applies to patients with type 2 diabetes. Although the UK study did not find a higher risk of breast cancer mortality in insulintreated patients with type 2 diabetes (based on an age of onset of 30-49 years) [15], such an assertion of a lack of association in patients with type 2 diabetes is questionable for the following reasons: (1) the sample was distorted and not representative of type 2 diabetes because only insulintreated patients were selected; (2) the diagnosis based on age of onset of diabetes was not reliable; and (3) there were a relatively small number of cases-only 2,122 insulintreated diabetic women with the asserted diagnosis of type 2 diabetes, and among these, only 17 cases of breast cancer mortality.

The postulated mechanisms linking diabetes and breast cancer are activation of insulin pathway, activation of the insulin-like growth factor pathway and regulation of endogenous sex hormones [2, 22]. Although we were not able to clarify the mechanisms linking diabetes and breast cancer, our results did provide strong evidence for a link in the Taiwanese population. 
Increased leptin and decreased adiponectin levels have been observed in individuals with obesity, type 2 diabetes and the metabolic syndrome, which may also contribute to the increased risk of breast cancer, especially the more aggressive cancers [23, 24]. Breast cancer patients with diabetes were more likely to die (RR 1.76 (95\% CI $1.23-$ 2.52) in one study [25]), suggesting that the prognosis for breast cancer was poorer in the diabetic patients than in those without diabetes. We did not have any biochemical data on our patients and were therefore unable to evaluate how biochemical parameters affected the mortality rates. The significantly higher mortality rate ratios observed in the present study or in studies evaluating breast cancer mortality should not be misinterpreted as significantly higher incidence rate ratios.

The strengths of this study include that it is a prospective follow-up study of a large cohort of diabetic patients, the probable completeness of ascertainment of vital status by obtaining the computerised data file for each individual from the National Register of Deaths using their unique identification number, and the exclusion of possible cases of type 1 diabetes to demonstrate a close link with type 2 diabetes. Nevertheless, there are some limitations. We evaluated cancer mortality and not incidence in this study. If diabetes has an effect on the case fatality rate, then estimation of the mortality rate ratio may not properly reflect the incidence rate ratio. There may be an increased detection bias in the diabetic patients because they may have visited their physicians more frequently, rendering a higher probability of detecting the existence of cancers. However, this may only suggest a higher detection rate of early and slowly progressive breast cancer with a better prognosis in the diabetic patients, which probably would have attenuated the mortality rate. Diabetic patients may have taken more medications than those without diabetes, which may have complicated the situation. For example, metformin, a widely used oral glucose-lowering agent, has been shown to afford protection against breast cancer [26, 27]. Therefore, if patients were taking metformin, this would underestimate the risk of breast cancer associated with diabetes. We were not able to evaluate the effects of medications because of a lack of data; the NHI did not allow access to this information and details were not requested in the baseline questionnaire because most patients are unable to correctly name the medications they use. In the calculation of the mortality rates in the diabetic patients, the person-year denominators for those who were alive were calculated from the date of recruitment until the end of the year 2006 without considering censoring, at the date of emigration, those who emigrated (not available for the study). This may have led to an overestimation of the person-year denominators of the mortality rates and underestimated the mortality rates for the diabetic patients.
However, the effect of emigration would be expected to be small because the emigration rate for the total population ranged from 0.05 to $0.60 \%$ for the female population over the period of the study according to the yearly report of the Ministry of the Interior of Taiwan [28]. Furthermore, the underestimation of the mortality rates in the diabetic patients could only have underestimated the mortality rate ratios. Therefore, the conclusions of the present study would not be affected.

In conclusion, we have demonstrated an increasing trend of mortality from breast cancer in all age groups in Taiwanese women over the 12 year period between 1995 and 2006. Furthermore, the risk of mortality from breast cancer is increased in diabetic patients, and the magnitude of the risk is largest in the younger age group of 25-54 years, in contrast to a more commonly observed association in postmenopausal women in the Western world. Given that the population is ageing and the incidence of type 2 diabetes is increasing, especially in the younger generation in Taiwan [19], the affect of breast cancer on mortality should warrant public health attention.

Acknowledgements The authors thank T.-T. Chan for providing excellent help with the statistical analyses and the following institutes for their continuous support of the epidemiological studies of diabetes mellitus and arsenic-related health hazards: the New Century Health Care Promotion Foundation, the Department of Health (DOH89-TD1035; DOH97-TD-D-113-97009), the National Taiwan University Hospital Yun-Lin Branch (NTUHYL96.G001) and the National Science Council of Taiwan (NSC-86-2314-B-002-326, NSC-872314-B-002-245, NSC88-2621-B-002-030, NSC89-2320-B002-125, NSC-90-2320-B-002-197, NSC-92-2320-B-002-156, NSC-93-2320B-002-071, NSC-94-2314-B-002-142, NSC-95-2314-B-002-311 and NSC-96-2314-B-002-061-MY2).

Duality of interest The authors declare that there is no duality of interest associated with this manuscript.

\section{References}

1. Freund E (1885) Zur diagnose des carcinoms. Wien Med B1:268268 (in German)

2. Xue F, Michels KB (2007) Diabetes, metabolic syndrome, and breast cancer: a review of the current evidence. Am J Clin Nutr $86: 823 \mathrm{~S}-835 \mathrm{~S}$

3. Larsson SC, Mantzoros CS, Wolk A (2007) Diabetes mellitus and risk of breast cancer: a meta-analysis. Int J Cancer 121:856-862

4. Muti P, Quattrin T, Grant BJ et al (2002) Fasting glucose is a risk factor for breast cancer: a prospective study. Cancer Epidemiol Biomark Prev 11:1361-1368

5. Rapp K, Schroeder J, Klenk J et al (2006) Fasting blood glucose and cancer risk in a cohort of more than 140,000 adults in Austria. Diabetologia 49:945-952

6. Barclay AW, Petocz P, McMillan-Price J et al (2008) Glycemic index, glycemic load, and chronic disease risk - a meta-analysis of observational studies. Am J Clin Nutr 87:627-637

7. Rollison DE, Giuliano AR, Sellers TA et al (2008) Populationbased case-control study of diabetes and breast cancer risk in 
Hispanic and non-Hispanic White women living in US southwestern states. Am J Epidemiol 167:447-456

8. Wu AH, Yu MC, Tseng CC, Stanczyk FZ, Pike MC (2007) Diabetes and risk of breast cancer in Asian-American women. Carcinogenesis 28:1561-1566

9. Goodman MT, Cologne JB, Moriwaki H, Vaeth M, Mabuchi K (1997) Risk factors for primary breast cancer in Japan: 8-year follow-up of atomic bomb survivors. Prev Med 26:144-153

10. Jee SH, Ohrr H, Sull JW, Yun JE, Ji M, Samet JM (2005) Fasting serum glucose level and cancer risk in Korean men and women. JAMA 293:194-202

11. Khan M, Mori M, Fujino Y, Japan Collaborative Cohort Study Group et al (2006) Site-specific cancer risk due to diabetes mellitus history: evidence from the Japan Collaborative Cohort (JACC) Study. Asian Pac J Cancer Prev 7:253-259

12. Inoue M, Iwasaki M, Otani T, Sasazuki S, Noda M, Tsugane S (2006) Diabetes mellitus and the risk of cancer: results from a large-scale population-based cohort study in Japan. Arch Intern Med 166:1871-1877

13. Coughlin SS, Calle EE, Teras LR, Petrelli J, Thun MJ (2004) Diabetes mellitus as a predictor of cancer mortality in a large cohort of US adults. Am J Epidemiol 159:1160-1167

14. Verlato G, Zoppini G, Bonora E, Muggeo M (2003) Mortality from site-specific malignancies in type 2 diabetic patients from Verona. Diabetes Care 26:1047-1051

15. Swerdlow AJ, Laing SP, Qiao Z et al (2005) Cancer incidence and mortality in patients with insulin-treated diabetes: a UK cohort study. Br J Cancer 92:2070-2075

16. Tseng CH (2004) Mortality and causes of death in a national sample of diabetic patients in Taiwan. Diabetes Care 27:16051609

17. Tseng $\mathrm{CH}$ (2006) Prevalence of lower-extremity amputation among patients with diabetes mellitus: Is height a factor? CMAJ 174:319-323
18. Tseng CH (2006) Exogenous insulin use and hypertension in adult patients with type 2 diabetes mellitus. Arch Intern Med 166:1184 1189

19. Tseng CH, Tseng CP, Chong CK et al (2006) Increasing incidence of diagnosed type 2 diabetes in Taiwan: analysis of data from a national cohort. Diabetologia 49:1755-1760

20. Tseng CH (2007) Parental history of hypertension and diabetes and prevalence of hypertension in type 2 diabetic patients: A study of a national sample in Taiwan. Eur J Clin Invest 37:870877

21. American Cancer Society (2006) Breast cancer facts and figures 2005-2006. American Cancer Society, Atlanta

22. Wolf I, Sadetzki S, Catane R, Karasik A, Kaufman B (2005) Diabetes mellitus and breast cancer. Lancet Oncol 6:103-111

23. Vona-Davis L, Howard-McNatt M, Rose DP (2007) Adiposity, type 2 diabetes and the metabolic syndrome in breast cancer. Obes Rev 8:395-408

24. Wolf I, Sadetzki S, Gluck I et al (2006) Association between diabetes mellitus and adverse characteristics of breast cancer at presentation. Eur J Cancer 42:1077-1082

25. Yancik R, Wesley MN, Ries LA, Havlik RJ, Edwards BK, Yates JW (2001) Effect of age and comorbidity in postmenopausal breast cancer patients aged 55 years and older. JAMA 285:885892

26. Zakikhani M, Dowling R, Fantus IG, Sonenberg N, Pollak M (2006) Metformin is an AMP kinase-dependent growth inhibitor for breast cancer cells. Cancer Res 66:10269-10273

27. Dowling RJ, Zakikhani M, Fantus IG, Pollak M, Sonenberg N (2007) Metformin inhibits mammalian target of rapamycindependent translation initiation in breast cancer cells. Cancer Res 67:10804-10812

28. Department of Statistics, Ministry of the Interior of Taiwan. Available from http://www.moi.gov.tw/stat/, accessed 18 September 2008 (in Chinese) 